\title{
Assessment of the prognostic value of interim fluorodeoxyglucose positron emission tomography/computed tomography in nasal-type extranodal natural killer/T-cell lymphoma
}

\author{
Xi Chen ${ }^{1 \# \wedge}$, Shengnan Zhao ${ }^{1 \#}$, Hongxi Wang ${ }^{2}$, Chunxi Fu ${ }^{1}$, Rong Tian ${ }^{2}$, Liqun Zou ${ }^{1 \wedge}$ \\ ${ }^{1}$ Department of Medical Oncology, Cancer Center, West China Hospital of Sichuan University, Chengdu, China; ${ }^{2}$ Department of Nuclear Medicine, \\ West China Hospital of Sichuan University, Chengdu, China
}

\#These authors contributed equally to this work.

Correspondence to: Liqun Zou. Department of Medical Oncology, Cancer Center, West China Hospital of Sichuan University, 37 Guoxue Alley, Wuhou District, Chengdu 610041, China. Email: zliqun@hotmail.com.

Background: The prognostic value of interim positron emission tomography/computed tomography (PET/ CT) for nasal-type extranodal natural killer/T-cell lymphoma (ENKTL) is controversial. We evaluated the prognostic value of interim PET/CT in ENKTL patients to facilitate risk stratification and guide clinical treatment.

Methods: Patients with ENKTL who received first-line chemotherapy based on L-asparaginase/ pegaspargase with/without involved-field radiotherapy were recruited for this study. Pretreatment and interim PET/CT evaluations were performed. Interim PET/CT was evaluated via the maximum standardized uptake value (SUVmax) and the Deauville 5-point scale (DS); and the capacity to predict progression-free survival (PFS) and overall survival (OS) was evaluated. Receiver operating characteristic (ROC) curves were used to determine the optimal SUVmax cutoff. Fisher's exact test was used to analyze relationships between interim PET/CT results and clinical characteristics. Univariate and multivariate analyses were performed to examine the independent effects of interim PET/CT. The Cochran-MantelHaenszel test was used to assess the prognostic value of interim PET/CT at different timepoints.

Results: Overall, 129 ENKTL patients were enrolled. The optimal interim PET/CT SUVmax cut-off was 4.95. The median follow-up was 34 [2-90] months, in the low SUVmax group ( $\leq 4.95)$, the 2-year PFS and OS rates were $76.3 \%$ and $88.0 \%$, respectively; in the high SUVmax group (>4.95), the PFS and OS rates were $15.6 \%$ and $44.5 \%$, respectively. Likewise, for the DS $1-3$ group, the PFS and OS rates were $78.9 \%$ and $91.2 \%$, respectively; and in the DS 4 or 5 group, the rates of PFS and OS were $49.7 \%$ and $69.0 \%$, respectively. In univariate analysis, interim PET/CT evaluation based on SUVmax and DS scores were both PFS and OS predictors. In multivariate analysis, SUVmax was independently significantly associated with PFS $(\mathrm{P}<0.001)$ and OS $(\mathrm{P}=0.002)$, and DS was independently significantly associated with $\mathrm{PFS}(\mathrm{P}=0.004)$ but not OS $(\mathrm{P}=0.204)$. In the Cochran-Mantel-Haenszel testing, the SUVmax and DS were significantly associated with PFS and OS after adjustments for the interim PET/CT timing.

Conclusions: Interim PET/CT was of prognostic value concerning ENKTL. The SUVmax is an independent prognostic indicator of PFS and OS, while the DS is an independent prognostic indicator of PFS but not OS. The SUVmax is of greater prognostic value than DS.

Keywords: Deauville 5-point scale (DS); interim positron emission tomography/computed tomography (interim PET/CT); nasal-type extranodal natural killer/T-cell lymphoma (ENKTL); prognostic value; maximum standardized uptake value (SUVmax)

^ ORCID: Xi Chen, 0000-0001-5398-3854; Liqun Zou, 0000-0003-4430-914. 
Submitted Apr 27, 2020. Accepted for publication Oct 23, 2020.

doi: 10.21037/qims-20-620

View this article at: http://dx.doi.org/10.21037/qims-20-620

\section{Introduction}

Nasal-type extranodal natural killer/T-cell lymphoma (ENKTL) is a subtype of invasive non-Hodgkin's lymphoma associated with Epstein-Barr virus (EBV) infection. In the World Health Organization classification, it is categorized as a mature T-cell and natural killer (NK) cell lymphoma subtype (1), and it is predominant in Asia and South America $(2,3)$. It is the second most common type of lymphoma in China after diffuse large B-cell lymphoma (DLBCL), and accounts for approximately $11 \%$ of all lymphomas. The average age at onset is 43.1 years, and the male-to-female ratio is approximately 2.6:1 (3).

There is currently no standardized treatment strategy for ENKTL. In the early stage (I/II), radiotherapy or combined chemoradiotherapy are mainly used, while in the advanced stage (III/IV), multi-drug combined chemotherapy is mainly applied. The prognosis of ENKTL patients has improved in recent years with the development of radiotherapy technology and the application of L-asparaginase or pegaspargase. In early stage patients, 5-year progressionfree survival (PFS) rates of 55.9-85.7\% have been reported, as have 5 -year overall survival (OS) rates of $78.6-89.0 \%$ $(4,5)$. However, some patients respond poorly to treatment, therefore, thorough evaluation of the efficacy of ENKTL treatments is warranted.

Many studies have shown that positron emission tomography/computed tomography (PET/CT) is more sensitive and accurate than enhanced CT, and that it can reveal hidden lesions $(6,7)$. The detection of such lesions can negate the underestimation of lymphoma stage and impact the management decisions in approximately a quarter of non-Hodgkin's lymphoma patients and a third of Hodgkin's lymphoma patients (7). In the initial staging, efficacy evaluation, prognostic evaluation, and recurrence monitoring of lymphoma, PET/CT have been widely used, particularly in DLBCL and Hodgkin's lymphoma $(8,9)$. The prognostic value of interim PET/CT differs for different types of lymphomas.

The prognostic value of interim PET/CT is controversial in DLBCL. Casasnovas et al. (10) and Yang et al. (11) have reported that it had useful prognostic value, whereas Pregno et al. (12) and Yoo et al. (13) reported that it did not, and that positive imaging did not predict a poor prognosis. The prognostic value of interim PET/CT in patients with Hodgkin's lymphoma has been confirmed in several studies, and thus it has become widely recognized (14-16). Studies have also indicated that ENKTLs tend to exhibit high fluorodeoxyglucose $\left({ }^{18} \mathrm{~F}\right.$-FDG) uptake $(17,18)$, which has resulted in ${ }^{18} \mathrm{~F}$-FDG PET/CT becoming one of the recommended examinations in relevant patients. Many studies have shown that pre-treatment (19-22) and post-treatment $(19,21,23) \mathrm{PET} / \mathrm{CT}$ have prognostic value concerning ENKTL, whereas fewer studies have investigated the prognostic value of interim PET/CT. Most studies have been retrospective investigations with small sample sizes, and they have included inconsistent evaluation parameters and have consequently yielded inconsistent conclusions $(19,21,24-27)$. Some studies have used the anthracycline-based cyclophosphamide, doxorubicin, vincristine, and prednisone (CHOP) regimen or a CHOP-like regimen rather than the currently recognized chemotherapy regimen based on L-asparaginase or pegaspargase (26). Therefore, the prognostic value of interim PET/CT in ENKTL is still uncertain.

In the current study, the prognostic value of interim PET/CT in ENKTL was analyzed retrospectively to establish a basis for clinical stratification and individualized treatment.

\section{Methods}

\section{Participants and treatment}

This retrospective study was conducted using medical records in the clinical database of the Center of Medical Oncology, West China Hospital, China. Patients aged 16-80 years who had been newly diagnosed with ENKTL as defined by the 2008 World Health Organization classification system (28) at the hospital between September 2010 and May 2017 were screened for enrolment. Exclusion criteria were as follows: (I) age $<16$ or $>80$ years; (II) lesions had been removed, or there were no evaluable lesions; (III) diagnosis of primary cutaneous ENKTL; (IV) chemotherapy regimen did not include L-asparaginase or pegaspargase; (V) presence of another malignant disease; or (VI) pregnancy or lactation.

All patients underwent staging procedures, including 
clinical and laboratory tests and bone marrow biopsies, and received chemotherapy and/or involved-field radiotherapy. Combined chemoradiotherapy patients received a 'sandwich' protocol, with 2-3 cycles of inductive chemotherapy, then radiotherapy was initiated, followed by 1-4 'consolidation' cycles of chemotherapy, totaling 4-6 cycles. All chemotherapy was based on L-asparaginase or pegaspargase, mainly the VDLP regimen (etoposide $75 \mathrm{mg} / \mathrm{m}^{2}$ on day $1-3$, dexamethasone $40 \mathrm{mg}$ on day $1-4$, L-asparaginase $5,000 \mathrm{IU} / \mathrm{m}^{2}$ on day $1-5$ or pegaspargase $2,500 \mathrm{IU} / \mathrm{m}^{2}$ on day 4 , and cisplatin $25 \mathrm{mg} / \mathrm{m}^{2}$ on day $1-3$, administered every 3 weeks), or the LVP regimen (L-asparaginase $5,000 \mathrm{IU} / \mathrm{m}^{2}$ on day $1-5$ or pegaspargase $2,500 \mathrm{IU} / \mathrm{m}^{2}$ on day 1 , vincristine $1.4 \mathrm{mg} / \mathrm{m}^{2}$, max $2 \mathrm{mg}$ on day 1 , prednisone $100 \mathrm{mg}$ on day $1-5$, administered every 3 weeks). The radiotherapy utilized incorporated intensitymodulated radiation therapy or three-dimensional (3D) conformal radiation therapy and conventional segmentation (1.8-2.0 Gy each administration) once per day, 5 times a week. The total radiation dose associated with radiotherapy was 50-56 Gy. Some participants received synchronous cisplatin chemotherapy on day $1-3$ every 3 weeks during radiotherapy, which was $25 \mathrm{mg} / \mathrm{m}^{2}$. All participants underwent PET/CT before and during the treatment. At 3-4 weeks after 2 or 3 cycles of chemotherapy or radiotherapy, all patients were evaluated via $\mathrm{PET/CT}$.

\section{PET/CT acquisition}

All patients underwent whole-body ${ }^{18} \mathrm{~F}$-FDG PET/CT performed using a combined Gemini GXL PET/CT scanner with a 16-slice CT component (Philips Corp., Amsterdam, Netherlands). After $6 \mathrm{~h}$ of fasting (no oral or intravenous fluids containing sugar or dextrose), 185$370 \mathrm{MBq}$ of ${ }^{18} \mathrm{~F}$-FDG $(5.18 \mathrm{MBq} / \mathrm{kg})$ was administered intravenously. The participant's blood glucose level was checked immediately before the ${ }^{18} \mathrm{~F}-\mathrm{FDG}$ administration. The PET/CT scanning was rescheduled if the blood glucose level was $>150 \mathrm{mg} / \mathrm{dL}$. Whole-body PET/CT scans from the top of the head to the feet were performed $60 \mathrm{~min}$ after radiopharmaceutical injection. Low-dose whole-body spiral CT was performed first, with parameters including $120 \mathrm{kV}, 40 \mathrm{mAs}$, layer thickness $5 \mathrm{~mm}$, and pitch 0.938 . The PET images were obtained in 3D mode, 16-18 beds were scanned regularly, and emission data were acquired for $2 \mathrm{~min}$ in each bed position. The CT acquisition data were used for attenuation correction, and corrected PET images were reconstructed using the ordered subsets expectation maximization method. The images acquired from the PET and CT scans were sent for image registration and fusion using Syntegra software (Philips Corp., Amsterdam, Netherlands).

\section{PET analysis}

The PET images were visually interpreted collaboratively by two experienced nuclear medicine physicians. The maximum standardized uptake value (SUVmax) and the Deauville 5-point scale (DS) were used to evaluate PET/CT results. Regions of interest (ROIs) were drawn manually along the edges of lesions, and the SUVs of ROIs were obtained automatically. The SUVmax was defined as the uptake in the brightest pixel in the ROI. A positive result was defined as any region with a metabolic increase that was not commensurate with the normal anatomical structure or normal variation. The DS compared the uptake of radionuclides in the region of focus with the participant's own mediastinal blood pool and liver as follows: 1 , no uptake above background; 2, uptake $\leq$ the mediastinum; 3 , uptake $>$ the mediastinum but $\leq$ the liver; 4 , uptake moderately $>$ the liver; 5, uptake markedly $>$ the liver and/ or the appearance of new lesions appearing; "X", uptake detected at a new site, and uptake not considered to be related to lymphoma.

\section{Efficacy evaluation}

Efficacy was evaluated following the 2014 Lugano evaluation standard (29), with PFS and OS as the main endpoints. The definition of PFS was the time from diagnosis to disease recurrence, progression, or diseaserelated death. Definition of OS was the time from diagnosis to death due to disease, or the last day of follow-up.

\section{Statistical analysis}

The software SPSS version 21.0 (SPSS Inc., Chicago, IL, USA) was used for statistical analysis. Receiver operating characteristic (ROC) curves were used to determine the best cutoff value of PFS of interim PET/CT SUVmax, and the participants were divided into two groups based on the consensus of the First International Symposium on PET/CT evaluation of mid-term lymphoma (30); a group with interim PET/CT DS scores of 1-3, and another with interim PET/CT DS scores of 4 or 5. Fisher's exact test was used to analyze relationships between interim PET/ 
CT results and clinical characteristics. Kaplan-Meier analysis and the log-rank test were used for univariate survival analysis. Cox proportional risk modeling was used to conduct multivariate analysis. The influences of PET/CT on the prediction of PFS and OS before and after radiotherapy were assessed using the CochranMantel-Haenszel test. Any P values $<0.05$ were considered statistically significant. Odds ratios (ORs) and confidence intervals (CIs) were calculated.

\section{Results}

\section{Patient characteristics}

A total of 129 patients were enrolled in the study between September 2010 and May 2017. The general participant characteristics are summarized in Table 1 . The median age was 45 years, $79(61.2 \%)$ were men, 105 (81.4\%) were diagnosed at stage I/II, and $24(18.6 \%)$ were diagnosed at stage III/IV. So-called B symptoms (weight loss, night sweats, and fever) were present in $70(54.3 \%)$ participants, $116(90.0 \%)$ had Eastern Cooperative Oncology Group (ECOG) performance status scores of 0 or 1 , and 102 (79.1\%) had Prognostic Index for Natural Killer Cell Lymphoma (PINK) scores (31) of 0 or 1 . Copy number testing of EBV DNA was conducted in 98/129 (76.0\%) participants before treatment, of which 75/98 (76.5\%) were positive and the other $23 / 98(23.5 \%)$ were negative or below the lower limit of detection $(<1.00 \mathrm{E}+03)$. Some 73 patients $(56.6 \%)$ underwent sequential chemoradiotherapy, $47(36.4 \%)$ underwent concurrent chemoradiotherapy, and $9(7.0 \%)$ underwent chemotherapy alone.

\section{Relationships between interim PET/CT results and clinical indices}

The overall median SUVmax was 3.90 (1.60-14.80). In the non-progression group, it was 3.50 (1.60-6.90), in the progression group it was $5.06(1.80-14.80)$, in the surviving group it was $3.70(1.60-11.00)$, and in the deceased group, it was 4.85 (1.80-14.80). In ROC curve analysis, the optimal SUVmax cutoff was 4.95 [sensitivity (Se) $51.9 \%$, specificity (Sp) 94.7\%, area under the curve (AUC) 0.750]. Based on these results, the patients were divided into a low SUVmax group $(\leq 4.95, \mathrm{n}=97)$ and a high SUVmax group $(>4.95, \mathrm{n}=32)$. The SUVmax and treatment outcomes are shown in Table 2. In the low SUVmax group, 26 patients $(26.8 \%)$ exhibited disease progression or died, and in the high SUVmax group, 28 (87.5\%) patients exhibited disease progression or died. In chi-square $\left(\chi^{2}\right)$ testing, high SUVmax was significantly associated with disease progression or death $(\mathrm{P}<0.001)$.

There were 51 participants in the DS $1-3$ group, and treatment failed in $11(21.6 \%)$ of these patients. There were 78 participants in the DS 4 or 5 group, and treatment failed in $43(55.1 \%)$ of these patients (Table 2). In $\left(\chi^{2}\right)$ testing, being assigned a DS score of 4 or 5 was significantly associated with treatment failure $(\mathrm{P}<0.001)$.

Patients were divided into groups based on interim PET/ CT SUVmax and DS scores, and relationships between clinical characteristics and SUVmax and DS scores were assessed using Fisher's exact test (Table 1). The invasion of regional lymph nodes was significantly associated with DS $(\mathrm{P}<0.001)$ and SUVmax of interim PET/CT $(\mathrm{P}=0.016)$. Regional lymph node invasion was associated with higher DS scores and SUVmax. Stage $(\mathrm{P}=0.003)$, B symptoms $(\mathrm{P}=0.040)$, number of extranodal invasion sites $(\mathrm{P}=0.008)$, and PINK score $(\mathrm{P}=0.001)$ were associated with SUVmax. Stage III/IV, B symptoms, $>2$ extranodal invasion sites, and PINK scores $\geq 2$ were associated with higher SUVmax.

\section{Survival analysis}

The median duration of follow-up was 34 [2-90] months. The median PFS and OS of the whole patient group and the low SUVmax group were not reached. In the high SUVmax group, the median PFS time was 3 months, and the median OS time was 20 months. In the low SUVmax group, the 2-year PFS rate was $76.3 \%$, and in the high SUVmax group, it was $15.6 \%$ (Figure $1 A$ ). The 2 -year OS rate in the low SUVmax group was $88.0 \%$, and $44.5 \%$ in the high SUVmax group (Figure 1B). Median PFS and OS in the DS 1-3 group were not achieved. The median PFS in the DS 4 or 5 group was 24 months, and the median OS was not reached. The 2 -year PFS rate in the DS $1-3$ group was $78.9 \%$, and was $49.7 \%$ in the DS $4-5$ group (Figure 1C). The 2-year OS rate in the DS $1-3$ group was $91.2 \%$, and $70.6 \%$ in the DS $4-5$ group (Figure 1D). The PET/CT SUVmax was significantly associated with PFS $(\mathrm{P}<0.001)$ and $\mathrm{OS}(\mathrm{P}<0.001)$, and interim PET/CT DS was also significantly associated with PFS $(\mathrm{P}<0.001)$ and $\mathrm{OS}(\mathrm{P}=0.010)$.

The patients were divided into two groups: a PINK score 0 or 1 group (low/medium risk), and a PINK score $\geq 2$ groups (high risk). The 2-year PFS rate in the PINK score 0 or 1 group was $69.7 \%$, and $29.6 \%$ in the PINK score $\geq 2$ groups $(\mathrm{P}<0.001)$ (Figure $1 E)$. The 2 -year $\mathrm{OS}$ rate in the 
Table 1 Relationships between PET/CT parameters and clinical characteristics

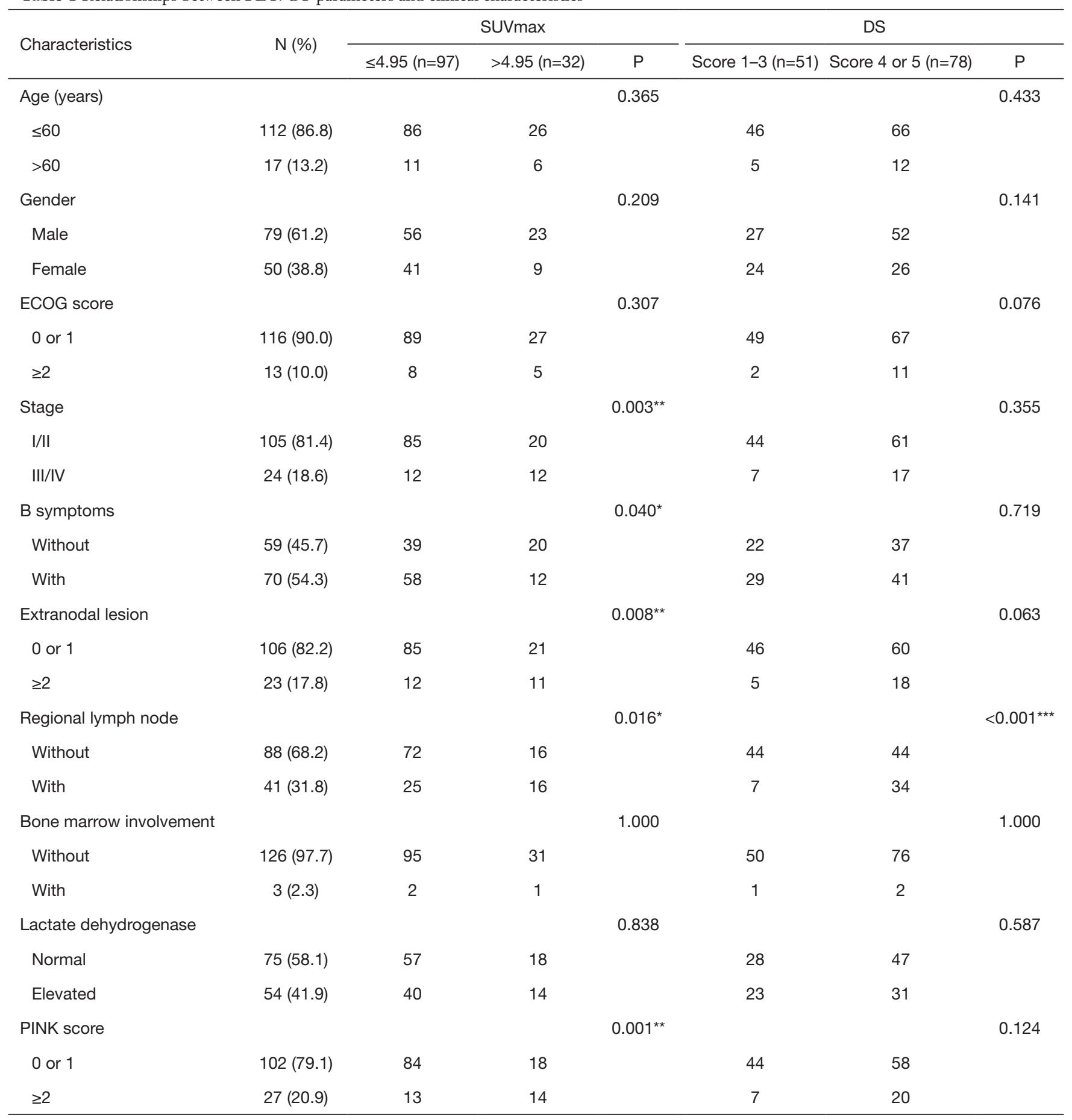

*, $\mathrm{P}<0.05 ;{ }^{* \star}, \mathrm{P}<0.01 ;{ }^{* \star *}, \mathrm{P}<0.001$. PET/CT, positron emission tomography/computed tomography; SUVmax, maximum standardized uptake value; DS, Deauville 5-point scale; ECOG, Eastern Cooperative Oncology Group; PINK, Prognostic Index of Natural Killer Cell Lymphoma. 
Table 2 Associations between treatment outcomes and interim PET/CT SUVmax and DS score

\begin{tabular}{|c|c|c|c|c|}
\hline Groups & \multicolumn{2}{|c|}{ SUVmax } & \multicolumn{2}{|c|}{ DS } \\
\hline Progression/death, n (\%) & $26(26.8)$ & $28(87.5)$ & $11(21.6)$ & $43(55.1)$ \\
\hline No progression, n (\%) & $71(73.2)$ & $4(12.5)$ & $40(78.4)$ & 35 (44.9) \\
\hline Total, $\mathrm{n}$ & 97 & 32 & 51 & 78 \\
\hline
\end{tabular}

PET/CT, positron emission tomography/computed tomography; SUVmax, maximum standardized uptake value; DS, Deauville 5-point scale.
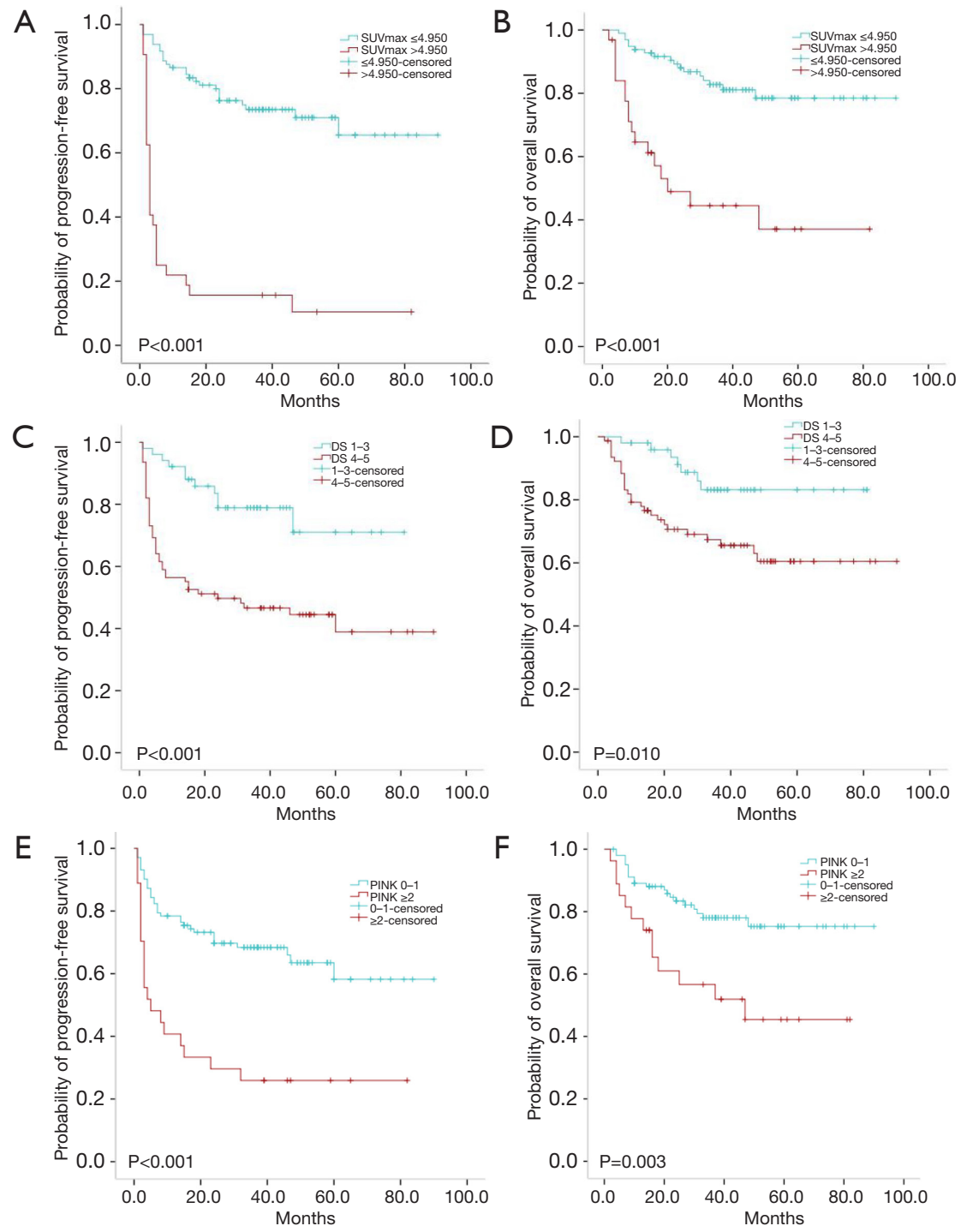

Figure 1 Survival analysis based on interim SUVmax, DS or PINK score. (A) PFS and (B) OS in ENKTL patients estimated using interim SUVmax during treatment; (C) PFS and (D) OS outcomes in ENKTL patients based on interim PET/CT status interpreted using the DS; (E) PFS and (F) OS outcomes in ENKTL patients based on the PINK score. PFS, progression-free survival; OS, overall survival; ENKTL, nasal-type extranodal natural killer/T-cell lymphoma; SUVmax, maximum standardized uptake value; PET/CT, positron emission tomography/computed tomography; DS, Deauville 5-point scale; PINK, Prognostic Index for Natural Killer Cell Lymphoma. 
PINK score 0 or 1 group was $83.4 \%$, and $61.0 \%$ in the PINK score $\geq 2$ groups $(\mathrm{P}=0.003$ ) (Figure $1 F)$.

\section{Univariate analysis}

Results of univariate survival analysis of numerous factors that may have affected ENKTL patients' prognosis are shown in Table 3. Gender, ECOG score, stage, extent of extranodal involvement, and regional lymph node involvement were all associated with PFS and OS. Being female, having an ECOG score of 0 or 1, being diagnosed as stage I/II, having <2 extranodal sites, and having no regional lymph node involvement were all associated with better PFS and OS. However, age $>60$ years, B-symptoms, and lactic acid dehydrogenase (LDH) levels were not significantly associated with PFS and OS.

\section{Multivariate analysis}

Considering that there may be some overlap between SUVmax and DS scores, and that the DS score 2-4 sometimes needed to be corrected by the SUVmax (30), the two parameters were analyzed separately. The results of multivariate analysis of interim PET/CT SUVmax are shown in Table 4. Gender $(\mathrm{P}=0.020$, OR: $0.453,95 \%$ CI: $0.232-0.883)$ and PET/CT SUVmax $(\mathrm{P}<0.001$, OR: 0.172, 95\% CI: 0.095-0.312) were independent predictors of PFS. Female participants and those with a SUVmax $\leq 4.95$ were more likely to exhibit better PFS. Gender ( $\mathrm{P}=0.011$, OR: 0.314, 95\% CI: 0.128-0.771), ECOG score $(\mathrm{P}=0.019$, OR: 0.313 , 95\% CI: 0.118-0.829), and PET/CT SUVmax ( $\mathrm{P}=0.002$, OR: 0.301, 95\% CI: 0.142-0.638) were independently associated with OS, and females, participants with ECOG scores $<2$, and participants with SUVmax $\leq 4.95$ had better OS. Interim PET/CT SUVmax and gender were independent predictors of PFS and OS, and ECOG was an independent predictor of OS.

In the multivariate analysis based on interim PET/ CT DS evaluation, gender $(\mathrm{P}=0.016$, OR: $0.455,95 \%$ CI: $0.240-0.862)$ and PET/CT DS score $(\mathrm{P}=0.004$, OR: $0.352,95 \%$ CI: $0.171-0.722)$ were independent prognostic factors of PFS; and female patients with DS scores of 1-3 had better PFS (Table 5). Gender ( $\mathrm{P}=0.005$, OR: 0.279, 95\% CI: $0.115-0.674)$ was an independent predictor of OS, and female participants had better OS. Interim PET/ CT DS score ( $\mathrm{P}=0.204$, OR: 0.551, 95\% CI: 0.220-1.382) was not an independent predictor of OS. Gender was an independent predictor of PFS and OS, and the DS score was an independent predictor of PFS but not OS.

Because PINK scores were significantly associated with PFS and OS in univariate analyses, multivariate analysis was conducted based on the PINK score. The Cox multivariate analysis results, including the PINK score and gender, ECOG score, number of extranodal sites, regional lymph node involvement, and LDH level, are shown in Table 6. Gender ( $\mathrm{P}=0.003$, OR: 0.385, 95\% CI: 0.204-0.726) was an independent predictor of PFS, and females had better PFS. The PINK score ( $\mathrm{P}=0.199$, OR: 0.483, 95\% CI: 0.160 1.464) was not an independent predictor of PFS. Gender $(\mathrm{P}=0.002$, OR: $0.250,95 \%$ CI: 0.104-0.599) and the ECOG score $(\mathrm{P}=0.042$, OR: $0.393,95 \%$ CI: $0.159-0.968)$ were independent predictors of OS, and females and participants with ECOG scores $<2$ had better OS. The PINK score $(\mathrm{P}=0.869$, OR: $1.131,95 \%$ CI: $0.263-4.853)$ was not an independent OS predictor.

\section{Comparison and consistency of the prognostic capacities of SUVmax and DS}

The PET/CT SUVmax had high Sp (94.7\%), high positive predictive value (PPV) $(87.5 \%)$, and a high positive likelihood ratio (PLR) (9.72) concerning the prediction of PFS, with an accuracy of $76.7 \%$. For the prediction of OS, the Sp of PET/CT SUVmax was $84.2 \%$; its negative predictive value (NPV) was $82.5 \%$, PPV was $53.1 \%$, Se was $50.0 \%$, and its accuracy was $75.2 \%$. The $\mathrm{Sp}$ of interim PET/CT DS concerning predicting PFS was $79.6 \%$, and its NPV was $78.4 \%$, but its accuracy was only $64.3 \%$. In a kappa consistency test of the two parameters, the kappa value was 0.355 .

\section{Stratification analysis}

The Cochran-Mantel-Haenszel test was used to assess the prognostic value of SUVmax and DS concerning ENKTL, based on whether PET/CT was performed before or after radiotherapy. After eliminating the influence of the timing of PET/CT, SUVmax and DS were significantly associated with PFS and OS (Table 7). Of 88 patients tested for EBV DNA copy number mid-treatment, 36 (40.9\%) were positive, and EBV DNA was negative or undetectable in the other $52(59.1 \%)$. In the positive group, 18/36 (50.0\%) had disease progression, and $10(27.8 \%)$ died. In the negative group, $14 / 52$ (26.9\%) had disease progression, and $6(11.5 \%)$ died. In the positive group, the 2-year PFS rate was $49.8 \%$, and the 2 -year OS rate was $73.8 \%$, and the 
Table 3 Univariate analysis of PFS and OS in ENKTL patients

\begin{tabular}{|c|c|c|c|c|}
\hline Characteristics & \multicolumn{2}{|c|}{ PFS } & \multicolumn{2}{|c|}{ OS } \\
\hline Age (years) & & 0.637 & & 0.779 \\
\hline$\leq 60$ & 61.7 & & 79.4 & \\
\hline$>60$ & 58.8 & & 75.5 & \\
\hline Male & 54.9 & & 69.9 & \\
\hline Female & 71.3 & & 89.7 & \\
\hline ECOG score & & $<0.001^{\star \star \star}$ & & $<0.001^{\star \star \star}$ \\
\hline 0 or 1 & 65.7 & & 82.6 & \\
\hline $\mathrm{I} / \mathrm{II}$ & 68.7 & & 83.9 & \\
\hline III/IV & 29.2 & & 51.9 & \\
\hline B symptoms & & 0.505 & & 0.729 \\
\hline Without & 57.0 & & 76.4 & \\
\hline With & 64.9 & & 79.0 & \\
\hline Extranodal lesion & & $<0.001^{\star \star \star}$ & & $<0.001^{\star \star \star}$ \\
\hline 0 or 1 & 69.0 & & 84.0 & \\
\hline$\geq 2$ & 26.1 & & 49.7 & \\
\hline Elevated & 53.1 & & 74.2 & \\
\hline PINK score & & $<0.001^{\star \star \star}$ & & $0.003^{\star \star}$ \\
\hline 0 or 1 & 69.7 & & 83.4 & \\
\hline$\geq 2$ & 29.6 & & 61.0 & \\
\hline SUVmax & & $<0.001^{\star \star *}$ & & $<0.001^{\star \star \star}$ \\
\hline$\leq 4.95$ & 76.3 & & 88.0 & \\
\hline$>4.95$ & 15.6 & & 44.5 & \\
\hline DS & & $<0.001^{\star \star \star}$ & & $0.010^{*}$ \\
\hline $1-3$ & 78.9 & & 91.2 & \\
\hline 4 or 5 & 49.7 & & 70.6 & \\
\hline
\end{tabular}

*, $\mathrm{P}<0.05$; ${ }^{* *}, \mathrm{P}<0.01 ;{ }^{* \star *}, \mathrm{P}<0.001$. PFS, progression-free survival; OS, overall survival; ENKTL, nasal-type extranodal natural killer/T-cell lymphoma; ECOG, Eastern Cooperative Oncology Group; LDH, lactic acid dehydrogenase; PINK, Prognostic Index of Natural Killer Cell Lymphoma; SUVmax, maximum standardized uptake value; DS, Deauville 5-point scale. 
Table 4 Multivariate analysis to assess associations between interim PET/CT SUVmax and PFS and OS

\begin{tabular}{|c|c|c|c|c|c|c|}
\hline Characteristics & \multicolumn{3}{|c|}{ PFS } & \multicolumn{3}{|c|}{ OS } \\
\hline Gender & 0.453 & $0.232-0.883$ & $0.020^{*}$ & 0.314 & $0.128-0.771$ & $0.011^{*}$ \\
\hline ECOG score & 0.782 & $0.310-1.970$ & 0.602 & 0.313 & $0.118-0.829$ & $0.019^{*}$ \\
\hline Stage & 0.911 & $0.215-3.855$ & 0.900 & 0.906 & $0.169-4.857$ & 0.908 \\
\hline Regional lymph node & 0.751 & $0.399-1.412$ & 0.374 & 0.651 & $0.301-1.407$ & 0.275 \\
\hline LDH & 0.759 & $0.418-1.377$ & 0.364 & 0.937 & $0.441-1.991$ & 0.865 \\
\hline SUVmax & 0.172 & $0.095-0.312$ & $<0.001^{\star * *}$ & 0.301 & $0.142-0.638$ & $0.002^{\star *}$ \\
\hline
\end{tabular}

*, $\mathrm{P}<0.05$; ${ }^{* *}, \mathrm{P}<0.01 ;{ }^{* \star *}, \mathrm{P}<0.001$. PET/CT, positron emission tomography/computed tomography; SUVmax, maximum standardized uptake value; PFS, progression-free survival; OS, overall survival; OR, odds ratio; CI, confidence interval; ECOG, Eastern Cooperative Oncology Group; LDH, lactic acid dehydrogenase.

Table 5 Multivariate analysis to assess associations between interim PET/CT DS score and PFS and OS

\begin{tabular}{|c|c|c|c|c|c|c|}
\hline Characteristics & \multicolumn{3}{|c|}{ PFS } & \multicolumn{3}{|c|}{ OS } \\
\hline Gender & 0.455 & $0.240-0.862$ & $0.016^{*}$ & 0.279 & $0.115-0.674$ & $0.005^{\star \star}$ \\
\hline ECOG score & 0.775 & $0.334-1.797$ & 0.552 & 0.410 & $0.166-1.016$ & 0.054 \\
\hline Stage & 0.422 & $0.107-1.663$ & 0.218 & 0.566 & $0.098-3.259$ & 0.524 \\
\hline Regional lymph node & 0.827 & $0.425-1.607$ & 0.574 & 0.660 & $0.286-1.526$ & 0.331 \\
\hline $\mathrm{LDH}$ & 0.698 & $0.389-1.250$ & 0.226 & 0.829 & $0.396-1.738$ & 0.620 \\
\hline DS score & 0.352 & $0.171-0.722$ & $0.004^{\star *}$ & 0.551 & $0.220-1.382$ & 0.204 \\
\hline
\end{tabular}

*, $\mathrm{P}<0.05$; ${ }^{*}, \mathrm{P}<0.01$. PET/CT, positron emission tomography/computed tomography; DS, Deauville 5-point scale; PFS, progression-free survival; OS, overall survival; OR, odds ratio; Cl, confidence interval; ECOG, Eastern Cooperative Oncology Group; LDH, lactic acid dehydrogenase.

Table 6 Multivariate analysis to assess associations between PINK score and PFS and OS

\begin{tabular}{|c|c|c|c|c|c|c|}
\hline Characteristics & \multicolumn{3}{|c|}{ PFS } & \multicolumn{3}{|c|}{ OS } \\
\hline Gender & 0.385 & $0.204-0.726$ & $0.003^{\star *}$ & 0.250 & $0.104-0.599$ & $0.002^{* *}$ \\
\hline ECOG score & 0.669 & $0.294-1.524$ & 0.339 & 0.393 & $0.159-0.968$ & $0.042^{*}$ \\
\hline Extranodal lesion & 0.748 & $0.242-2.308$ & 0.614 & 0.347 & $0.081-1.499$ & 0.156 \\
\hline Lactate dehydrogenase & 0.839 & $0.474-1.483$ & 0.546 & 0.945 & $0.457-1.950$ & 0.877 \\
\hline PINK score & 0.483 & $0.160-1.464$ & 0.199 & 1.131 & $0.263-4.853$ & 0.869 \\
\hline
\end{tabular}

*, $\mathrm{P}<0.05 ;{ }^{* *}, \mathrm{P}<0.01$. PINK, Prognostic Index of Natural Killer Cell Lymphoma; PFS, progression-free survival; OS, overall survival; OR, odds ratio; $\mathrm{Cl}$, confidence interval; ECOG, Eastern Cooperative Oncology Group. 
Table 7 Capacity of interim PET/CT SUVmax or DS scores before and after radiotherapy to predict PFS and OS

\begin{tabular}{|c|c|c|c|c|c|c|c|c|c|c|c|c|}
\hline \multirow{2}{*}{ PET/CT timing } & \multicolumn{6}{|c|}{ SUVmax } & \multicolumn{6}{|c|}{ DS } \\
\hline & OR & $95 \% \mathrm{Cl}$ & $\mathrm{P}$ & OR & $95 \% \mathrm{Cl}$ & $\mathrm{P}$ & OR & $95 \% \mathrm{Cl}$ & $\mathrm{P}$ & OR & $95 \% \mathrm{Cl}$ & $\mathrm{P}$ \\
\hline $\begin{array}{l}\text { Before radiotherapy } \\
\text { After radiotherapy }\end{array}$ & 0.056 & $0.017-0.189$ & $<0.001^{\star \star \star}$ & 0.186 & $0.066-0.529$ & $0.001^{\star \star}$ & 0.213 & $0.091-0.501$ & $<0.001^{\star \star \star}$ & 0.330 & $0.123-0.884$ & $0.022^{*}$ \\
\hline
\end{tabular}

*, $\mathrm{P}<0.05 ;{ }^{* \star}, \mathrm{P}<0.01$; ${ }^{* \star}, \mathrm{P}<0.001$. $\mathrm{PET} / \mathrm{CT}$, positron emission tomography/computed tomography; SUVmax, maximum standardized uptake value; DS, Deauville 5-point scale; PFS, progression-free survival; OS, overall survival; OR, odds ratio; $\mathrm{Cl}$, confidence interval.
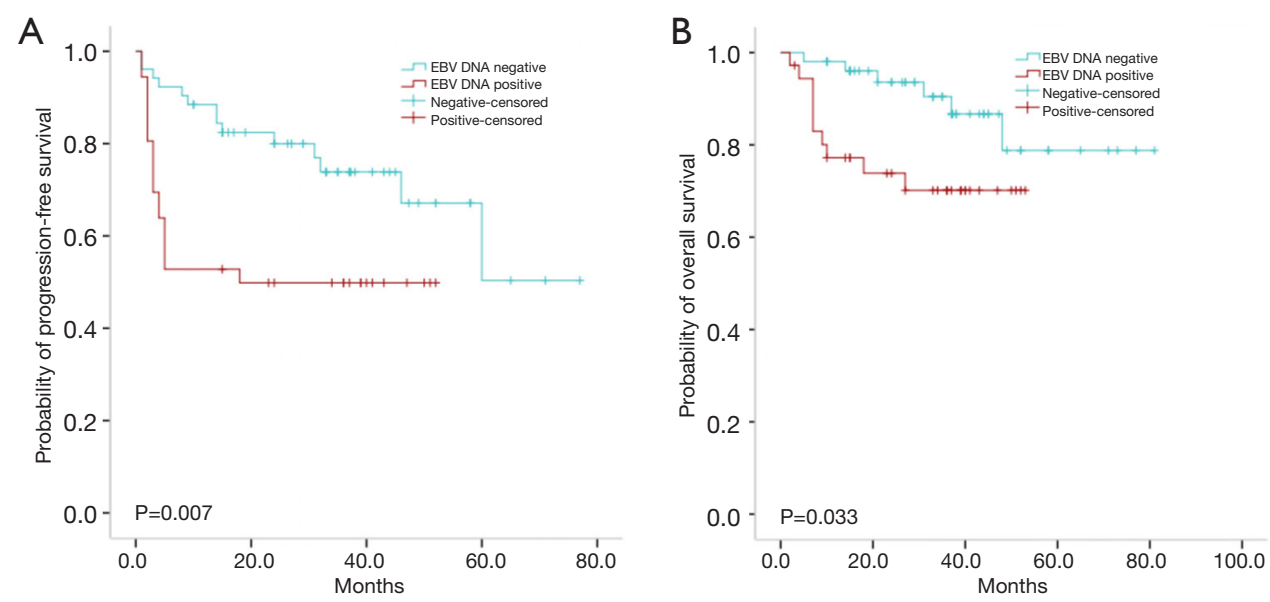

Figure 2 Survival analysis based on interim EBV DNA status. (A) PFS and (B) OS in ENKTL patients based on interim EBV DNA status. PFS, progression-free survival; OS, overall survival; ENKTL, nasal-type extranodal natural killer/T-cell lymphoma; EBV, Epstein-Barr virus.

corresponding rates in the negative group were $80.0 \%$ and $93.6 \%$, respectively. In univariate survival analysis, midtreatment EBV DNA status was significantly associated with PFS $(\mathrm{P}=0.007)$ and $\mathrm{OS}(\mathrm{P}=0.033$; Figure $2 A, B)$, but in Cox regression analysis mid-treatment EBV DNA status was not an independent predictor of PFS $(\mathrm{P}=0.054)$ or $\mathrm{OS}$ $(\mathrm{P}=0.210)$ (Table 8$)$.

\section{Discussion}

Different clinical studies have investigated a variety of indicators of the efficacy of interim PET/CT in lymphoma patients, mainly including the consensus of the international harmonization project, DS, SUVmax, and change in SUVmax ( $\triangle$ SUVmax). In the current study, SUVmax and the DS were used to analyze the efficacy of interim PET/ $\mathrm{CT}$, and the results were consistent with the conclusions of most previous studies. Interim PET/CT was predictive of PFS and OS in ENKTL patients. The SUVmax was independently predictive of PFS and OS, whereas the DS was independently predictive of PFS but not of OS, which is not concordant with previous studies $(20,24,25)$.

The PET/CT SUVmax is a semi-quantitative analysis index that is easy to determine, and was significantly associated with PFS and OS in the present study. Khong et al. (25) conducted a retrospective analysis including 24 ENKTL patients, of which 19 were primary, and 5 had relapsed. In their study, the patients were divided into a low SUVmax group $(<3)$ and a high SUVmax group $(>3)$, and in multivariate analysis, SUVmax was not an independent predictor of PFS or OS. The differences in findings between that study and the current study may be due to the small sample size in Khong et al. (25) and relapsed patients' inclusion in their study. In the present study, an interim PET/CT-determined SUVmax $\leq 4.95$ was predictive of better PFS and OS, and an interim SUVmax $>4.95$ predicted poorer PFS but the not poorer OS, as SUVmax had poor PPV for the prediction of OS. Patients 
Table 8 Multivariate analysis to assess associations between interim EBV DNA status and PFS and OS

\begin{tabular}{|c|c|c|c|c|c|c|}
\hline Characteristics & \multicolumn{3}{|c|}{ PFS } & \multicolumn{3}{|c|}{ OS } \\
\hline Gender & 0.586 & $0.253-1.357$ & 0.212 & 0.715 & $0.219-2.335$ & 0.578 \\
\hline ECOG score & 0.471 & $0.137-1.624$ & 0.233 & 0.207 & $0.045-0.952$ & $0.043^{*}$ \\
\hline Stage & 0.363 & $0.064-2.046$ & 0.251 & 0.281 & $0.034-2.335$ & 0.240 \\
\hline Regional lymph node & 0.793 & $0.364-1.728$ & 0.560 & 0.839 & $0.297-2.368$ & 0.740 \\
\hline LDH & 0.687 & $0.307-1.539$ & 0.362 & 0.919 & $0.274-3.086$ & 0.891 \\
\hline EBV DNA & 0.470 & $0.218-1.013$ & 0.054 & 0.474 & $0.147-1.524$ & 0.210 \\
\hline
\end{tabular}

*, P<0.05. EBV, Epstein-Barr virus; PFS, progression-free survival; OS, overall survival; OR, odds ratio; Cl, confidence interval; ECOG, Eastern Cooperative Oncology Group; LDH, lactic acid dehydrogenase.

with an interim PET/CT SUVmax $>4.95$ may require a stronger chemotherapy regimen, an increased number of chemotherapy cycles, or the administration of new drug treatments such as immunotherapy; these patients should also be closely monitored for recurrence after treatment.

The DS is currently internationally recommended and widely used in interim PET/CT evaluation (30), and it was used in the current study to investigate the prognostic value of interim PET/CT in ENKTL patients. In Cox regression analysis, the DS was an independent predictor of PFS but not of OS. Jiang et al. (24) conducted a prospective study including 60 ENKTL patients using DS to evaluate the prognostic value of interim PET/CT, and DS was found to be an independent predictor of PFS and OS in that study. Chang et al. (21) and Khong et al. (25) both reached the same conclusion as Jiang et al. (24). The DS is a widely recommended method for evaluating the efficacy of PET/ CT in lymphoma patients, but it exhibited unsatisfactory prognostic value in the present study. Possible reasons for this include the fact that divergent treatment regimens have been used in different studies, as have varied PET/CT parameters, and there are also differences in the timing of interim PET/CT between individual studies. There can be remaining tumor residues in some patients with large tumor loads and good initial responses to treatment. Lastly, there is a prolonged inflammatory period at the radiotherapy site, and this inflammation causes increased ${ }^{18}$ F-FDG uptake. Therefore, the PET/CT may be associated with a high false-positive rate in the middle stage after radiotherapy, which may affect the interpretation of results.

In the present study, PET/CT was of the same prognostic value before or after radiotherapy, but this needs to be verified in a clinical trial with larger sample size. Notably, PET/CT was able to identify patients exhibiting poor treatment efficacy in the early stage, but it is not clear whether the timely adjustment of treatment based on interim PET/CT results would yield survival benefits. The $\Delta$ SUVmax was investigated in the multicenter, prospective, randomized, controlled "PETAL" study (31), which included 76 T-cell non-Hodgkin's lymphoma patients. Of these participants, 21 had anaplastic lymphoma kinase (ALK)-positive anaplastic large cell lymphoma, 13 had ALK-negative anaplastic large cell lymphoma, 18 had angioimmunomaternal T-cell lymphoma, and 20 had peripheral T-cell lymphoma, not otherwise specified. The patients were divided into two groups based on a $\triangle \mathrm{SUV}$ max cut-off of $66 \%$, and there were $57(75 \%)$ patients in the $>66 \%$ group and $19(25 \%)$ in the $\leq 66 \%$ group. The 2 -year OS rates were $79 \%$ in the $>66 \%$ group and $25 \%$ in the $\leq 66 \%$ group ( $\mathrm{P}=0.0001)$. Also, in participants in the $\leq 66 \%$ group, instigating a stronger chemotherapy regimen (methotrexate + cytarabine + etoposide) resulted in increased toxicity but did not improve the long-term effects of treatment compared with completing a CHOP regimen. Therefore, it is believed that PET/CT can identify patients with poor prognoses in the early stage, but that instigating a stronger chemotherapy regimen in these patients will not reliably be of benefit concerning survival. It may be necessary to consider introducing new types of drugs or targeted treatments to improve their prognoses. Notably, patients with ENKTL were not included in the PETAL study (31), and to date, no previously conducted prospective study is available to guide ENKTL treatment planning based on the results of interim PET/CT. Therefore, whether 
replacement therapy confers survival benefits in ENKTL patients who exhibit poor treatment efficacy as determined via interim PET/CT remains unknown. Whether the increased toxicity associated with the use of stronger chemotherapy can be controlled and if the introduction of new types of drugs and/or immunotherapies can confer benefits also warrants further investigation.

The PINK prediction model is widely used in the prognosis evaluation of ENKTL patients receiving nonanthracycline chemotherapy (32). In this study, we evaluated the prognostic significance of the PINK score in all patients and attempted to establish a prognosis model combining PET/CT and PINK scores. In the multivariate analysis conducted in the current study, the PINK score was not an independent predictor of PFS $(\mathrm{P}=0.199)$ or OS $(\mathrm{P}=0.869)$, which is not concordant with previous studies. These discrepancies may be related to the small number of cases in the present study.

There is a strong association between ENKTL and EBV infection, and previous studies have suggested that EBV DNA in circulation can reflect tumor load and predict prognoses and curative effects (32-34). In the present study, EBV DNA was significantly associated with PFS and OS in univariate analysis, but in multivariate analysis, it was not a significant independent predictor of PFS $(\mathrm{P}=0.054)$ or OS $(\mathrm{P}=0.210)$. Notably, mid-treatment EBV DNA status was not tested in all patients in the current study, and EBV DNA status was simply categorized as positive or negative and included in the analysis. Associations between EBV DNA and PFS and OS in ENKTL patients warrant further research and verification.

Compared with other studies, the current study had several advantages. To the best of our knowledge, it is the largest investigation of the prognostic value of interim PET/CT in ENKTL patients concerning sample size. The first-line chemotherapy was based on L-asparaginase or pegaspargase, and $93 \%$ of the participants underwent involved-field radiotherapy, which is currently recognized as a more effective treatment. All patients were evaluated via SUVmax and DS, and DS is the recommended interim PET/CT evaluation method.

Our study also had some limitations. The sample size was comparatively small, and some of the laboratory data were incomplete. Some patients did not undergo pre-treatment and mid-treatment EBV DNA assessment, meaning that PINK and PINK with EBV DNA (so-called "PINK-E") scores could not be calculated, and the prognostic value EBV DNA status could not be accurately analyzed. Thus, the results need to be verified in further prospective randomized studies involving large-scale multicenter collaborative groups.

In conclusion, interim PET/CT exhibited prognostic value in the ENKTL patients in the current study. Interim PET/CT SUVmax was an independent predictor of PFS and OS, while interim PET/CT DS was a predictor of PFS but not OS. The interim PET/CT SUVmax had higher accuracy for PFS and OS than the DS score for PFS. Our study suggests that the SUVmax value is of greater prognostic value than the DS and warrants inclusion in the clinical evaluation of ENKTL patients.

\section{Acknowledgments}

Funding: None.

\section{Footnote}

Conflicts of Interest: All authors have completed the ICMJE uniform disclosure form (available at http://dx.doi. org/10.21037/qims-20-620). The authors have no conflicts of interest to declare.

Ethical Statement: The study was conducted in accordance with the Declaration of Helsinki (as revised in 2013). This study was approved by the Biomedical Research Ethics Committee of West China Hospital of Sichuan University (2020733).

Open Access Statement: This is an Open Access article distributed in accordance with the Creative Commons Attribution-NonCommercial-NoDerivs 4.0 International License (CC BY-NC-ND 4.0), which permits the noncommercial replication and distribution of the article with the strict proviso that no changes or edits are made and the original work is properly cited (including links to both the formal publication through the relevant DOI and the license). See: https://creativecommons.org/licenses/by-nc-nd/4.0/.

\section{References}

1. Swerdlow SH, Campo E, Pileri SA, Harris NL, Stein H, Siebert R, Advani R, Ghielmini M, Salles GA, Zelenetz AD, Jaffe ES. The 2016 revision of the World Health Organization classification of lymphoid neoplasms. Blood 2016;127:2375-90.

2. Tse E, Kwong YL. How I treat NK/T-cell lymphomas. 
Blood 2013;121:4997-5005.

3. Sun J, Yang Q, Lu Z, He M, Gao L, Zhu M, Sun L, Wei L, Li M, Liu C, Zheng J, Liu W, Li G, Chen J. Distribution of lymphoid neoplasms in china analysis of 4,638 cases according to the World Health Organization Classification. Am J Clin Pathol 2012;138:429-34.

4. Bi XW, Xia Y, Zhang WW, Sun P, Liu PP, Wang Y, Huang JJ, Jiang WQ, Li ZM. Radiotherapy and PGEMOX/ GELOX regimen improved prognosis in elderly patients with early-stage extranodal NK/T-cell lymphoma. Ann Hematol 2015;94:1525-33.

5. Dong LH, Zhang LJ, Wang WJ, Lei W, Sun X, Du JW, Gao X, Li GP, Li YF. Sequential DICE combined with l-asparaginase chemotherapy followed by involved field radiation in newly diagnosed, stage IE to IIE, nasal and extranodal NK/T-cell lymphoma. Leuk Lymphoma 2016;57:1600-6.

6. Elstrom RL, Leonard JP, Coleman M, Brown RK. Combined PET and low-dose, noncontrast CT scanning obviates the need for additional diagnostic contrastenhanced CT scans in patients undergoing staging or restaging for lymphoma. Ann Oncol 2008;19:1770-3.

7. Raanani P, Shasha Y, Perry C, Metser U, Naparstek E, Apter S, Nagler A, Polliack A, Ben-Bassat I, Even-Sapir E. Is CT scan still necessary for staging in Hodgkin and nonHodgkin lymphoma patients in the PET/CT era? Ann Oncol 2006;17:117-22.

8. Juweid ME, Stroobants S, Hoekstra OS, Mottaghy FM, Dietlein M, Guermazi A, Wiseman GA, Kostakoglu L, Scheidhauer K, Buck A, Naumann R, Spaepen K, Hicks RJ, Weber WA, Reske SN, Schwaiger M, Schwartz LH, Zijlstra JM, Siegel BA, Cheson BD. Use of positron emission tomography for response assessment of lymphoma: consensus of the Imaging Subcommittee of International Harmonization Project in Lymphoma. J Clin Oncol 2007;25:571-8.

9. Cheson BD. Role of functional imaging in the management of lymphoma. J Clin Oncol 2011;29:1844-54.

10. Casasnovas RO, Meignan M, Berriolo-Riedinger A, Bardet $\mathrm{S}$, Julian A, Thieblemont C, Vera P, Bologna S, Brière J, Jais JP, Haioun C, Coiffier B, Morschhauser F. SUVmax reduction improves early prognosis value of interim positron emission tomography scans in diffuse large B-cell lymphoma. Blood 2011;118:37-43.

11. Yang DH, Min JJ, Song HC, Jeong YY, Chung WK, Bae SY, Ahh JS, Kim YK, Bom HS, Chung IJ, Kim HJ, Lee JJ. Prognostic significance of interim 18F-FDG PET/CT after three or four cycles of R-CHOP chemotherapy in the treatment of diffuse large B-cell lymphoma. Eur J Cancer 2011;47:1312-8.

12. Pregno P, Chiappella A, Bellò M, Botto B, Ferrero S, Franceschetti S, Giunta F, Ladetto M, Limerutti G, Menga M, Nicolosi M, Priolo G, Puccini B, Rigacci L, Salvi F, Vaggelli L, Passera R, Bisi G, Vitolo U. Interim 18-FDGPET/CT failed to predict the outcome in diffuse large B-cell lymphoma patients treated at the diagnosis with rituximab-CHOP . Blood 2012;119:2066-73.

13. Yoo C, Lee DH, Kim JE, Jo J, Yoon DH, Sohn BS, Kim SW, Lee JS, Suh C. Limited role of interim PET/CT in patients with diffuse large B-cell lymphoma treated with R-CHOP. Ann Hematol 2011;90:797-802.

14. Zinzani PL, Rigacci L, Stefoni V, Broccoli A, Puccini B, Castagnoli A, Vaggelli L, Zanoni L, Argnani L, Baccarani M, Fanti S. Early interim 18F-FDG PET in Hodgkin's lymphoma: evaluation on 304 patients. Eur J Nucl Med Mol Imaging 2012;39:4-12.

15. Cerci JJ, Pracchia LF, Linardi CC, Pitella FA, Delbeke D, Izaki M, Trindade E, Soares J Jr, Buccheri V, Meneghetti JC. 18F-FDG PET after 2 cycles of ABVD predicts eventfree survival in early and advanced Hodgkin lymphoma. J Nucl Med 2010;51:1337-43.

16. Hutchings M, Loft A, Hansen M, Pedersen LM, Buhl T, Jurlander J, Buus S, Keiding S, D'Amore F, Boesen AM, Berthelsen AK, Specht L. FDG-PET after two cycles of chemotherapy predicts treatment failure and progressionfree survival in Hodgkin lymphoma. Blood 2006;107:52-9.

17. Khong PL, Pang CB, Liang R, Kwong YL, Au WY. Fluorine-18 fluorodeoxyglucose positron emission tomography in mature T-cell and natural killer cell malignancies. Ann Hematol, 2008;87:613-21.

18. Chan WK, Au WY, Wong CY, Liang R, Leung AY, Kwong YL, Khong PL. Metabolic activity measured by F-18 FDG PET in natural killer-cell lymphoma compared to aggressive B- and T-cell lymphomas. Clin Nucl Med 2010;35:571-75

19. Jiang C, Zhang X, Jiang M, Zou L, Su M, Kosik RO, Tian $\mathrm{R}$. Assessment of the prognostic capacity of pretreatment, interim, and post-therapy (18)F-FDG PET/CT in extranodal natural killer/T-cell lymphoma, nasal type. Ann Nucl Med 2015;29:442-51.

20. Kim CY, Hong CM, Kim DH, Son SH, Jeong SY, Leo SW, Lee J, Ahn BC. Prognostic value of whole-body metabolic tumour volume and total lesion glycolysis measured on 18F-FDG PET/CT in patients with extranodal NK/T-cell lymphoma. Eur J Nucl Med Mol Imaging 2013;40:1321-9. 
21. Chang Y, Fu X, Sun Z, Xie X, Wang R, Li Z, Zhang $X$, Sheng G, Zhang M. Utility of baseline, interim and end-of-treatment 18F-FDG PET/CT in extranodal natural killer/T-cell lymphoma patients treated with L-asparaginase/pegaspargase. Sci Rep 2017;7:41057.

22. Ko KY, Liu CJ, Ko CL, Yen RF. Intratumoral heterogeneity of pretreatment 18F-FDG PET images predict disease progression in patients with nasal type extranodal natural killer/T-cell lymphoma. Clin Nucl Med 2016;41:922-6.

23. Jiang C, Liu J, Li L, Kosik RO, Su M, Zou L, Tian R. Predictive approaches for post-therapy PET/CT in patients with extranodal natural killer/T-cell lymphoma: a retrospective study. Nucl Med Commun 2017;38:937-47.

24. Jiang C, Su M, Kosik RO, Zou L, Jiang M, Tian R. The Deauville 5-point scale improves the prognostic value of interim FDG PET/CT in extranodal natural killer/T-cell lymphoma. Clin Nucl Med 2015;40:767-73.

25. Khong PL, Huang B, Lee EY, Chan WK, Kwong YL. Midtreatment 18F-FDG PET/CT scan for early response assessment of SMILE therapy in natural killer/T-cell lymphoma: a prospective study from a single center. J Nucl Med 2014;55:911-6.

26. Li YJ, Li ZM, Xia XY, Huang HQ, Xia ZJ, Lin TY, Li S, Xia Y, Cai XY, Jiang WQ. Prognostic value of interim and posttherapy 18F-FDG PET/CT in patients with mature T-cell and natural killer cell lymphomas. J Nucl Med 2013;54:507-15.

27. Qin C, Yang S, Sun X, Xia X, Li C, Lan X. 18F-FDG PET/CT for prognostic stratification of patients with extranodal natural killer/T-cell lymphoma. Clin Nucl Med 2019;44:201-8.

28. Campo E, Swerdlow SH, Harris NL, Pileri S, Stein H, Jaffe ES. The 2008 WHO classification of lymphoid neoplasms and beyond: evolving concepts and practical applications. Blood 2011;117:5019-32.

29. Cheson BD, Fisher RI, Barrington SF, Cavalli F, Schwartz LH, Zucca E, Lister TA; Alliance, Australasian Leukaemia and Lymphoma Group; Eastern Cooperative Oncology Group; European Mantle Cell Lymphoma Consortium; Italian Lymphoma Foundation; European Organisation for Research; Treatment of Cancer/Dutch Hemato-Oncology Group; Grupo Español de Médula Ósea; German HighGrade Lymphoma Study Group; German Hodgkin's Study Group; Japanese Lymphorra Study Group; Lymphoma Study Association; NCIC Clinical Trials Group; Nordic Lymphoma Study Group; Southwest Oncology Group; United Kingdom National Cancer Research Institute. Recommendations for initial evaluation, staging, and response assessment of Hodgkin and non-Hodgkin lymphoma: the Lugano classification. J Clin Oncol 2014;32:3059-68.

30. Meignan M, Gallamini A, Meignan M, Gallamini A, Haioun C. Report on the First International Workshop on interim-PET scan in lymphoma. Leuk Lymphoma 2009;50:1257-60.

31. Hüttmann A, Müller SP, Rekowski J, Hertenstein B, Franzius C, Franzke A, Bengel FM, Kroschinsky F, Kotzerke J, La Rosée P, Freesmeyer M, Hoeffkes HG, Hertel A, Behringer D, Mesters R, Weckesser M, Mahlmann S, Haberkorn U, Martens U, Prange-Krex G, Korfel A, Brenner W, Giagounidis A, Moeller R, Runde V, Sandmann M, Hautzel H, Wilop S, Krohn T, Dürk H, Strumberg D, Heike M, Claudia O, Brinkmann M, Trenn G, Kroke O, Kreisel-Büstgens C, Dang TA, Heil G, Jöckel KH, Hoelzer D, Klapper W, Duehrsen U. Positron emission tomography (PET) guided therapy of aggressive lymphomas - interim PET-based outcome prediction and treatment changes in patients with $\mathrm{T}$ cell lymphomas participating in the PETAL trial. Blood 2016;128:185.

32. Kim SJ, Yoon DH, Jaccard A, Chng WJ, Lim ST, Hong H, Park Y, Chang KM, Maeda Y, Ishida F, Shin DY, Kim JS, Jeong SH, Yang DH, Jo JC, Lee GW, Choi CW, Lee WS, Chen TY, Kim K, Jung SH, Murayama T, Oki Y, Advani R, d'Amore F, Schmitz N, Suh C, Suzuki R, Kwong YL, Lin TY, Kim WS. A prognostic index for natural killer cell lymphoma after non-anthracycline-based treatment: a multicentre, retrospective analysis. Lancet Oncol 2016;17:389-400.

33. Suzuki R, Yamaguchi M, Izutsu K, Yamamoto G, Takada K, Harabuchi Y, Isobe Y, Gomyo H, Koike T, Okamoto M, Hyo R, Suzumiya J, Nakamura S, Kawa K, Oshimi K. Prospective measurement of Epstein-Barr virusDNA in plasma and peripheral blood mononuclear cells of extranodal NK/T-cell lymphoma, nasal type. Blood 2011;118:6018-22.

34. Au WY, Pang A, Choy C, Chim CS, Kwong YL. Quantification of circulating Epstein-Barr virus (EBV) DNA in the diagnosis and monitoring of natural killer cell and EBV-positive lymphomas in immunocompetent patients. Blood 2004;104:243-9.

Cite this article as: Chen X, Zhao S, Wang H, Fu C, Tian R, Zou L. Assessment of the prognostic value of interim fluorodeoxyglucose positron emission tomography/computed tomography in nasal-type extranodal natural killer/T-cell lymphoma. Quant Imaging Med Surg 2021;11(4):1220-1233. doi: 10.21037/qims-20-620 